\title{
The Algebraic Structure of Spaces of Intervals Contribution of Svetoslav Markov to Interval Analysis and its Applications
}

\author{
Roumen Anguelov \\ Department of Mathematics and Applied Mathematics \\ University of Pretoria, Pretoria, South Africa \\ E-mail: roumen.anguelov@up.ac.za
}

In Interval Analysis addition of intervals is the usual Minkowski addition of sets: $A+B=\{a+b$ : $a \in A, b \in B\}$. The fact that the additive inverse generally does not exist has been a major obstacle in applications, e.g. constructing narrow enclosures of a solution, and possibly one of the most important mathematical challenges associated with the development of the theory of spaces of intervals. The work on this issue during the last 50-60 years lead to new operations for intervals, extended concepts of interval, setting the interval theory within the realm of algebraic structures more general than group and linear space. This theoretical development was paralleled by development of interval computer arithmetic. Svetoslav Markov was strongly involved in this major development in modern mathematics and he in fact introduced many of the main concepts and theories associated with it. These include: extended interval arithmetic [11], [5], [10], directed interval arithmetic [13], the theory of quasivector spaces [15], [16]. His work lead to practically important applications to the validated numerical computing as well as in the computations with intervals, convex bodies and stochastic numbers [12], [14], [18]. Such advanced mathematical and computational tools are much useful under the conditions of extreme sensitivity that is often inheritably characteristic for biological processes as well as input biological parameters experimentally known to be in certain ranges [17].

One of the most important contributions to knowledge by Svetoslav Markov is in my view the embedding of the monoid structure of intervals and convex bodies into a group structure where the natural definition of multiplication by scalars is also extended in such a way that it is monotone with respect to inclusion, that is

$$
A \subseteq B \Longrightarrow \gamma A \subseteq \gamma B, \quad \gamma \in \mathbb{R} .
$$

The obtained structure is called a quasivector space [16]. Since the fundamental idea motivating this field is computations with sets and computing enclosures, the stated property cannot be really overemphasized. One should note that there have been several attempts to embed the quasi-linear space of convex bodies in a more computationally convenient algebraic structure. The most well known such attempt is Radström's embedding into a linear space [19]. However, this embedding fails to preserve precisely the monotonicity property mentioned above. Hence, while the developed by Radström theory is mathematically correct and elegant, it is quite irrelevant regarding the embedded set and in fact the field of Interval Analysis or more generally the field of SetValued Computing. Markov's concept of quasivector space manages to capture and preserve the essential properties of computations with sets (like the stated monotonicity) while also providing a relatively simple structure for computing. Indeed, the quasivector space is a direct sum of a vector(linear) space and symmetric quasivector space which makes the computations essentially as easy as computations in a linear space.

A wide spectrum of applications is usually a testimony for the depth of an idea. The ideas of Markov have certainly wide and far reaching implications. We focus on one particular direction of development, namely the 
algebraic operations for interval-valued functions. I have had the privilege to have Svetoslav Markov as a teacher and as a collaborator. The algebraic structure of spaces of interval functions is the main topic of our more recent collaborative work. Jointly with Blagovest Sendov we studied the operations for Hausdorff continuous $(\mathrm{H}-$ continuous) function. It turned out that the linear space structure of real functions can be extended to the space of $\mathrm{H}$-continuous interval functions and it is actually the largest linear space of interval functions. Hence the space of $\mathrm{H}$-continuous functions has a very special place in Interval Analysis. Further, we showed that the practically relevant set, in terms of providing tight enclosures of sets of real functions, is the set of Dilworth continuous (D-continuous) interval valued function. Using an earlier idea of Svetoslav Markov of abstract construction of interval space over a vector lattice, one can show that the set of D-continuous function is a quasi-linear space of intervals over the space of $\mathrm{H}$-continuous functions. Moreover, the space of $\mathrm{H}$-continuous functions is precisely the linear space in the direct Markov's sum decomposition of the respective quasivector space.

Let us note that the space of $\mathrm{H}$-continuous functions has applications in various areas of mathematics, e.g. Real Analysis [1], [2], Approximation Theory [20], validated computing [3], [6] as well as the general theory of PDEs [9], [7], [4]. The issue of constructing enclosures is relevant to all mentioned applications. Hence one can expect future developments in this research direction which involve the space of D-continuous functions and Markov's approach to computing with them.

\section{REFERENCES}

[1] R Anguelov, Dedekind order completion of C(X) by Hausdorff continuous functions, Quaestiones Mathematicae, Vol. 27, 2004, pp. $153-170$.

[2] $\mathrm{R}$ Anguelov, Rational Extensions of $C(X)$ via Hausdorff Continuous Functions, Thai Journal of Mathematics, Vol. 5 No. 2, 2007, pp. 261-272.

[3] R Anguelov, Algebraic Computations with Hausdorff Continuous Functions, Serdica Journal of Computing, Vol. 1 No. 4, 2007, pp. 443-454.

[4] R Anguelov, D Agbebaku, J H van der Walt, Hausdorff Continuous Solutions of Conservation Laws, In MD Todorov (editor),Proceedings of the 4th International Conference on
Application of Mathematics in Technical and Natural Sciences (AMiTaNS'12), (St Constantine and Helena, Bulgaria), American Institute of Physics - AIP Conference Proceedings 1487, 2012, pp 151-158.

[5] R Anguelov R, S Markov, Extended Segment Analysis, Freiburger Intervall Berichte 81/10, University of Freiburg, 1981.

[6] R Anguelov, S Markov, Numerical Computations with Hausdorff Continuous Functions, In: T. Boyanov et al. (Eds.), Numerical Methods and Applications 2006 (NMA 2006), Lecture Notes in Computer Science 4310, Springer, 2007, 279-286.

[7] R Anguelov, S Markov, F Minani, Hausdorff Continuous Viscosity Solutions of Hamilton-Jacobi Equations, Proceedings of the 7th International Conference on Large Scale Scientific Computations, 3-8 June 2009, Sozopol, Bulgaria, Lecture Notes in Computer Science, 5910 (2010), pp. 241-248.

[8] R Anguelov, S Markov and Bl Sendov, The Set of Hausdorff Continuous Functions - the Largest Linear Space of Interval Functions, Reliable Computing, Vol. 12, 2006, pp. 337-363.

[9] R Anguelov, E E Rosinger, Solving Large Classes of Nonlinear Systems of PDE's, Computers and Mathematics with Applications, Vol. 53, 2007, pp. 491-507.

[10] Dimitrova, N., Markov, S., Popova, E., Extended Interval Arithmetics: New Results and Applications. In: Computer Arithmetic and Enclosure Methods (Eds. L. Atanassova, J. Herzberger), North-Holland, Amsterdam, 1992, 225-234.

[11] S Markov, A Non-standard Subtraction of Intervals, Serdica, 3, 1977, pp.359-370.

[12] S Markov, Calculus for interval functions of a real variable. Computing 22, 325-337 (1979).

[13] S Markov, On Directed Interval Arithmetic and its Applications, J. UCS 1 (7), 1995, 514-526.

[14] S Markov, An Iterative Method for Algebraic Solution to Interval Equations, Applied Numerical Mathematics 30 (2-3), 1999, 225-239.

[15] S Markov, On the Algebraic Properties of Convex Bodies and Some Applications, J. Convex Analysis 7 (1), 2000, 129-166.

[16] S Markov, On Quasilinear Spaces of Convex Bodies and Intervals, Journal of Computational and Applied Mathematics 162 (1), 93-112, 2004

[17] S Markov, Biomathematics and Interval Analysis: A Prosperous Marriage, in Ch Christov and MD Todorov (eds), Proceedings of the $2 \mathrm{~d}$ International Conference on Application of Mathematics in Technical and Natural Sciences (AMiTaNS'10), (21-26 June, Sozopol, Bulgaria), American Institute of Physics - AIP Conf. Proc. 1301, pp. 26-36.

[18] S Markov, R Alt, Stochastic Arithmetic: Addition and Multiplication by Scalars, Applied Numerical Mathematics 50, 475488, 2004.

[19] H Radström, An embedding theorem for spaces of convex sets. Proc. Am.Math. Soc. 3, 165-169. (1952)

[20] B1 Sendov, Hausdorff Approximations, Kluwer Academic, Boston, 1990. 